\title{
Effects of monoclinic symmetry on the properties of biaxial liquid crystals
}

\author{
Nikita V. Solodkov, Mamatha Nagaraj, and J. Cliff Jones* \\ School of Physics and Astronomy, University of Leeds, Leeds LS2 9JT, United Kingdom
}

(Received 13 August 2017; published 10 April 2018)

\begin{abstract}
Tilted smectic liquid crystal phases such as the smectic-C phase seen in calamitic liquid crystals are usually treated using the assumption of biaxial orthorhombic symmetry. However, the smectic-C phase has monoclinic symmetry, thereby allowing disassociation of the principal optic and dielectric axes based on symmetry and invariance principles. This is demonstrated here by comparing optical and dielectric measurements for two materials with highly first-order direct transitions from nematic to smectic-C phases. The results show a high difference between the orientations of the principal axes sets, which is interpreted as the existence of two distinct cone angles for optical and dielectric frequencies. Both materials exhibit an increasing degree of monoclinic behavior with decreasing temperature. Due to fast switching speeds, ferroelectric smectic- $\mathrm{C}^{*}$ materials are important for fast modulators and LCoS devices, where the dielectric biaxiality influences device operation.
\end{abstract}

DOI: 10.1103/PhysRevE.97.042702

\section{INTRODUCTION}

Symmetry leads to invariance principles and conservation laws, providing structure and coherence to the laws of nature [1]. The symmetry of a phase of matter is central to describing material physical properties, transitions between different phases, and the order parameters associated with those changes. Liquid crystals have a rich variety of mesophases with various symmetries. The most widely studied liquid crystal phases are the nematic $(\mathrm{N})$ and lamellar smectic-A (Sm-A) phases, in which the molecular anisotropy results in orientational order about the director $\mathbf{n}$ and a cylindrical symmetry of the phase, described by the $D_{\infty h}$ symmetry group [2]. Recently there has been much interest in the possibility of biaxial nematic phases and evidence for biaxial polar Sm-A found in certain bent-core liquid crystals [3-9]. The molecular bend angle in these systems further reduces the symmetry of phases they exhibit, leading to biaxial and polar mesophases even though the constituent molecules are achiral in nature. These phases may have triclinic or monoclinic symmetry $[6,10]$, but it is usually assumed that such phases have the highest symmetry $D_{2 h}$ and that the biaxial properties are orthorhombic. The goal of this work is to present experimental evidence for the effects of monoclinic symmetry of the Sm-C phase on the dielectric and optical properties of the phase. We demonstrate this by allowing a decoupling of the dielectric and optical properties while still satisfying the invariance principles of monoclinic symmetry. Additionally, we measure the extent of monoclinic behavior by interpreting it as a separation of cone angles for each observable.

In the smectic-C $(\mathrm{Sm}-\mathrm{C})$ phase, the director is tilted at the cone angle $\theta$ with respect to the layer normal a (see Fig. 1). McMillan proposed a model based on dipole-dipole interactions, where the torque generated by the outbound dipoles causes the molecules to tilt in the smectic-C phase [11].

\footnotetext{
*j.c.jones@leeds.ac.uk
}

A steric model was later proposed by Wulf, which considered the packing of symmetric molecules and suggested that a zigzag molecular shape is the primary driving mechanism in the breaking of the symmetry and formation of tilt within layers [12]. This suggests that, for a system to form a Sm-C phase, the aliphatic end chains of the molecules must be sufficiently long to induce a zigzag shape but insufficient to make the zigzag negligible. Since the molecules have monoclinic symmetry (described by the $C_{2 h}$ symmetry group), the $\mathrm{Sm}-\mathrm{C}$ phase is inherently biaxial. Understanding the symmetry properties of a simple $\mathrm{Sm}-\mathrm{C}$ system is a major stepping stone in understanding monoclinic symmetry in new noncalamitic liquid crystals.

When chirality is added to the Sm-C phase, the symmetry is reduced from $C_{2 h}$ to the $C_{2}$ group [13], thereby allowing for the appearance of the ferroelectric $\mathrm{Sm}-\mathrm{C}^{*}$ phase [14]. When compared to the dielectric torque used to switch N-based devices, the ferroelectric torque is usually much stronger, allowing much faster switching times at lower voltages. This is because ferroelectric liquid crystals exhibit a polar response to electric fields, which additionally allows both the "on" and and the "off" switching mechanisms to be driven electrically. For this reason, ferroelectric Sm-C* liquid crystals have been used in various applications [15-17]. Electro-optic behavior at high electric fields and frequencies beyond that of ferroelectric switching is dominated by the dielectric biaxiality $[17,18]$. Knowing the dielectric biaxiality is critical for understanding the behavior of devices, from fast electro-optical shutters to high-resolution liquid crystal on silicon spatial light modulators.

\section{MONOCLINIC SYMMETRY AND THE DIELECTRIC TENSOR}

Assuming orthorhombic symmetry between optical and permittivity axes allows the individual permittivity components to be calculated using the combination of optical and dielectric measurements. This requires permittivity measurements in planar and homeotropic geometries, $\varepsilon_{p}$ and $\varepsilon_{h}$, 
(a)

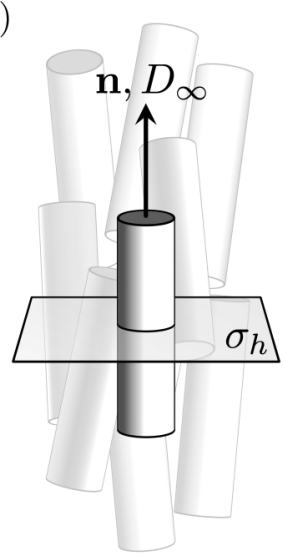

(b)

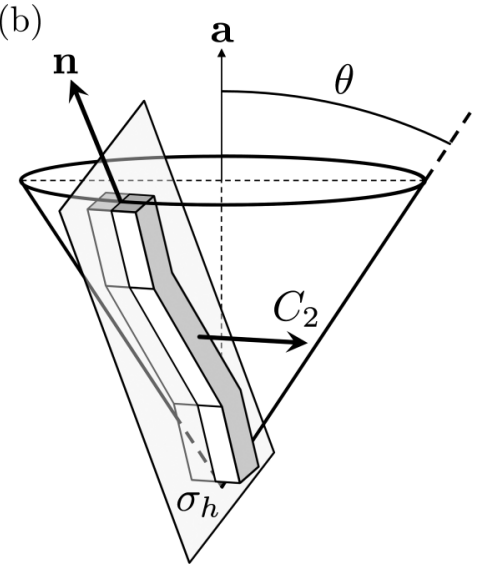

initially form with an in-plane tilt component in a planar device. The first-order transition leads to less layer shrinkage and correspondingly lower values of $\delta$. In such cases, asymptotic discontinuities arise whenever a $\mathrm{Sm}-\mathrm{C}$ material exhibits layer tilts which satisfy

$$
\delta \leqslant \delta_{\mathrm{c}}=\arcsin \left(|\sin \theta| \sqrt{\frac{\cos 2 \theta}{2-3 \sin ^{2} \theta}}\right),
$$

where $\delta_{c}$ is the critical layer tilt, below which equations described in Ref. [18] result in unphysical solutions. Measurements of the biaxial permittivities for materials with an $\mathrm{N}$ to $\mathrm{Sm}-\mathrm{C}$ phase sequence have not been reported before, partly because of this issue.

By the consideration of energy conservation and time

FIG. 1. Illustration defining the local symmetries of the (a) nematic and (b) smectic-C phases. The directors in both images are indicated with $\mathbf{n}$, the layer normal of the smectic-C phase by a, and the corresponding cone angle by $\theta . D_{\infty}$ represents the infinitefold rotational symmetry of the nematic phase, and $C_{2}$ represents the two-fold rotational symmetry of the smectic-C phase; both are accompanied by perpendicular mirror planes, $\sigma_{h}$.

respectively. Two similar methods were previously used to calculate the principal permittivity components in Sm- $\mathrm{C}^{*}$ materials: using electric fields to unwind the helical structure $[19,20]$ and using surface interactions in devices with sufficiently thin containment regions to permanently suppress the helix formation [18].

Since the Sm-C phase has monoclinic symmetry, the principal dielectric axes do not necessarily coincide with the optical axes. However, all previous measurements of the biaxial electric permittivities have used the optical cone angle $[18,20]$. The monoclinic order parameter will grow continuously from zero below the transition. Hence, the dispersion of the symmetry axes will also increase significantly. Thus, differences between the monoclinic physical properties may be more difficult to measure experimentally. By continuity principles, the monoclinic properties will appear gradually for second-order transitions (e.g., Sm-A to Sm-C), and therefore, monoclinic properties may not have a significant effect and lie within the experimental error. In such cases, an orthorhombic approximation is appropriate close to the phase transitions. However, if the phase transition is highly first order (e.g., $\mathrm{N}$ to $\mathrm{Sm}-\mathrm{C}$, where the biaxiality has a discontinuous jump), then we expect the monoclinic properties to have a more pronounced contribution, and monoclinic symmetry must be considered when studying dielectric properties of such materials.

As the sample is cooled into and through the Sm-C phase, the smectic layer spacing typically shrinks, resulting in the layers tilting to a chevron structure in planar geometry [21]. Typical materials that undergo the Sm-A to $\mathrm{Sm}-\mathrm{C}$ phase transition lead to a ratio of layer tilt $\delta$ to the cone angle $\theta$ that is roughly constant and around 0.85 [17]. This has been explained using the argument of monoclinic symmetry of the Sm-C $(*)$ phase, where the ratio corresponds to that of the mass and polarizability cone angles. However, in liquid crystals, which exhibit a first-order $\mathrm{N}$ to $\mathrm{Sm}-\mathrm{C}$ phase transition directly, layers reversal symmetry, it can be shown that the dielectric tensor $\tilde{\varepsilon}_{i j}$ is always symmetric [22,23]. Hence, we can use the spectral theorem for symmetric matrices, which states that a symmetric matrix is always orthogonally diagonalizable [24] such that

$\varepsilon_{i j}=\left(\begin{array}{ccc}\varepsilon_{1} & 0 & 0 \\ 0 & \varepsilon_{2} & 0 \\ 0 & 0 & \varepsilon_{3}\end{array}\right)=\left(\begin{array}{llll}\mathbf{u}_{1} & \mathbf{u}_{2} & \mathbf{u}_{3}\end{array}\right)^{\mathrm{T}} \tilde{\varepsilon}_{i j}\left(\begin{array}{lll}\mathbf{u}_{1} & \mathbf{u}_{2} & \mathbf{u}_{3}\end{array}\right)$,

where $\varepsilon_{k}$ are the eigenvalues and $\mathbf{u}_{k}$ are the eigenvectors of $\tilde{\varepsilon}_{i j}$, for $k=1,2,3$. The eigenvalues of the dielectric tensor are the principal dielectric permittivities, and the eigenvectors are the principal dielectric axes, such that $\mathbf{u}_{3}$ is the dielectric director. By applying invariance principles along the $C_{2}$ axis, we can deduce that there exists a principal dielectric axis $\varepsilon_{2}$ parallel to $C_{2}$, which coincides with analogous optic and other principal axes. The lack of orthorhombic symmetry results in a decoupling of the eigenvectors of permittivity and optics by rotating one with respect to another about the common $C_{2}$ axis. This asymmetry can be interpreted as a difference between the optical and dielectric cone angles

$$
\partial \theta=\theta_{o}-\theta_{\varepsilon},
$$

where $\theta_{o}$ and $\theta_{\varepsilon}$ are the cone angles generated by the optical and dielectric axes, respectively. Figure 2 illustrates the two cone angles with respect to the layer normal and indicates the principal optical and permittivity axes.

\section{GOVERNING EQUATIONS}

When measuring relative permittivities in the Sm-C phase in the laboratory frame of reference $(x, y, z)$ along the $(0,1,0)$ direction, one measures

$$
\varepsilon=\left(Q \varepsilon_{i j} Q^{\mathrm{T}}\right)_{y y},
$$

where $Q$ rotates the director by an angle $\theta$ away from $\mathbf{z}$, by an angle $\phi$ around $\mathbf{z}$, and by an angle $\delta$ around $\mathbf{x}$. For the general monoclinic case, $\partial \theta$ is not known and represents the difference between the optical and dielectric cone angles, $\theta_{o}$ and $\theta_{\varepsilon}$, respectively. The result of this is that the measured $\varepsilon$ consists of an orthorhombic part $\varepsilon^{o}$ (the case in which $\partial \theta=0$ ) with an additional monoclinic contribution, 


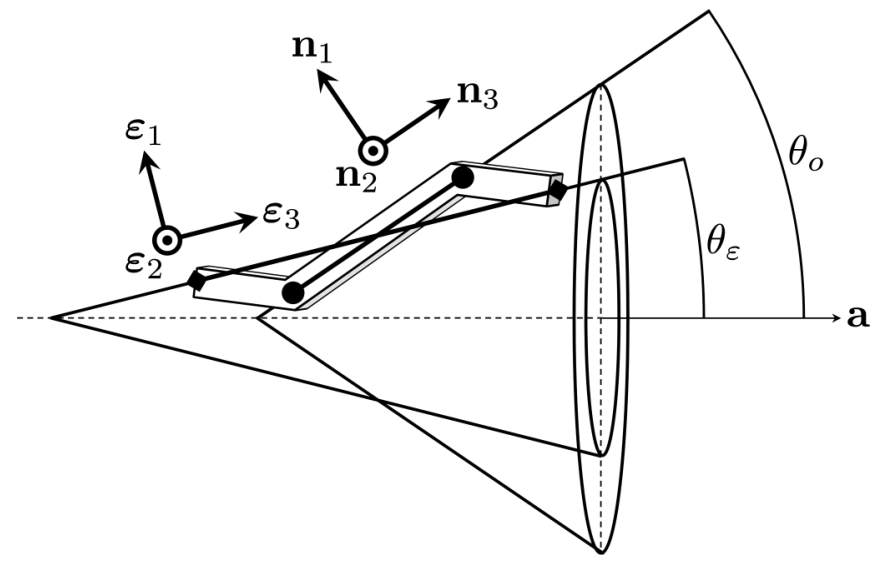

FIG. 2. Schematic representation of the assumed average molecular configuration of the Sm-C phase, illustrating the differences between the principal components of the optical and dielectric axes, indicated by $\mathbf{n}_{i}$ and $\boldsymbol{\varepsilon}_{i}$ (for $i=1,2,3$ ), respectively. The corresponding cone angles, measured from layer normal $\mathbf{a}$, are indicated by $\theta_{o}$ and $\theta_{\varepsilon}$.

given by

$$
\begin{gathered}
\varepsilon=\overbrace{\varepsilon_{11}+\left(\varepsilon_{2}-\varepsilon_{11}\right) \cos ^{2} \delta \cos ^{2} \phi+\left(\varepsilon_{33}-\varepsilon_{11}\right) \sin ^{2} \zeta}^{\text {orthorhombic }=\varepsilon^{o}} \\
\underbrace{+2 \varepsilon_{13} \sin \zeta(\cos \delta \sin \phi \cos \theta+\sin \delta \sin \theta)}_{\text {monoclinic correction }},
\end{gathered}
$$

where the out-of-plane director tilt, $\zeta$, is given by $\sin \zeta=$ $(\cos \delta \sin \phi \sin \theta-\sin \delta \cos \theta)$.

A rotation by an angle $\partial \theta$ around the $C_{2}$ axis of the phase diagonalizes the dielectric tensor. Therefore, we can choose a different cone angle to be $\theta_{\varepsilon}=\theta_{o}-\partial \theta$ and disassociate the other two principal dielectric and optical axes, reducing (4) to

$$
\varepsilon=\varepsilon_{1}+\left(\varepsilon_{2}-\varepsilon_{1}\right) \cos ^{2} \delta \cos ^{2} \phi+\left(\varepsilon_{3}-\varepsilon_{1}\right) \sin ^{2} \zeta_{\varepsilon},
$$

where $\zeta_{\varepsilon}$ now has $\theta=\theta_{\varepsilon}$, while $\delta$ and $\phi$ remain the same. Since we have chosen $\varepsilon_{2}$ to be in the direction of the $C_{2}$ axis of rotation, $\varepsilon_{2}$ and $\left(\varepsilon_{11}+\varepsilon_{33}\right)$ are invariant under any rotation about the $C_{2}$ axis.

If the material is ferroelectric, the director can be switched to the side of the cone in the planar geometry, and an additional permittivity measurement can be made by applying a dc bias to the sample [17]. In the fully switched position of the director (when the maximal amount of the $C_{2}$ axis points along the field, such that $\phi=0$ and $\zeta_{\varepsilon}=\zeta_{o}=\delta$ ) the measured permittivity is given by

$$
\varepsilon_{\mathrm{f}}=\varepsilon_{2} \cos ^{2} \delta+\varepsilon_{\mathrm{h}} \sin ^{2} \delta,
$$

where $\varepsilon_{p}$ and $\varepsilon_{h}$ are the permittivities measured in planar and homeotropic geometries, respectively. This allows $\varepsilon_{2}$ to be calculated directly and reduces the problem to the following three equations:

$$
\begin{gathered}
\varepsilon_{h}=\varepsilon_{1} \sin ^{2} \theta_{\varepsilon}+\varepsilon_{3} \cos ^{2} \theta_{\varepsilon}, \\
\varepsilon_{p}=\varepsilon_{1}\left(1-\cos ^{2} \delta \cos ^{2} \phi-\sin ^{2} \zeta_{\varepsilon}\right)+\varepsilon_{3} \sin ^{2} \zeta_{\varepsilon} \\
+\varepsilon_{2} \cos ^{2} \delta \cos ^{2} \phi \\
3 \bar{\varepsilon}=\varepsilon_{1}+\varepsilon_{2}+\varepsilon_{3},
\end{gathered}
$$

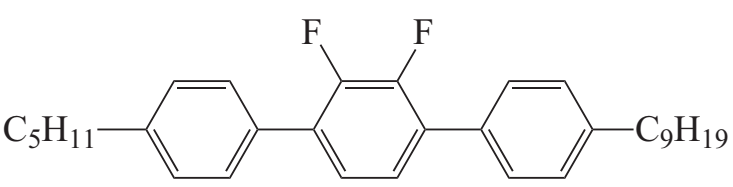

X: Cry 317 K Sm-C 339 K N 383 K I

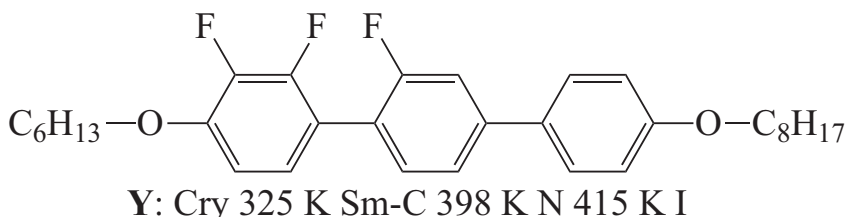

FIG. 3. Chemical structures for $\mathrm{X}\left[2^{\prime}, 3^{\prime}\right.$-difluoro-4-nonyl-4" pentyl-1, $1^{\prime}: 4^{\prime}, 1^{\prime \prime}$-terphenyl] and Y [2,2',3-trifluoro-4-(hexyloxy)-4'"-

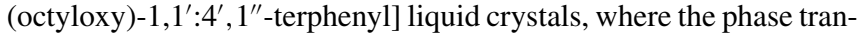
sition temperatures are shown under the corresponding structures.

where $\bar{\varepsilon}$ is the mean permittivity, which can be extrapolated from the uniaxial and isotropic phases. Equations (6)-(9) can be solved numerically to find a unique solution for the principal permittivities and the dielectric cone angle. Additionally, these equations remove the restriction due to the discontinuity for $\delta \leqslant \delta_{\mathrm{c}}$ and allow us to calculate relative permittivities for materials with low $\delta$ to $\theta$ ratios, such as those with direct $\mathrm{N}$ to Sm-C phase transitions.

\section{MATERIALS AND EXPERIMENTS}

In this work, we investigate the dielectric and optical properties of two partially fluorinated terphenyl liquid crystals with direct $\mathrm{N}$ to $\mathrm{Sm}-\mathrm{C}$ phase transitions, both of which potentially show highly monoclinic behavior. Compound X, 2', 3'-difluoro-4-nonyl-4"'-pentyl-1, 1' $: 4^{\prime}, 1^{\prime \prime}$ terphenyl [25], has a strong coupling between the sole dipole moment and the polarizability tensor. On the other hand, compound Y, 2,2',3-trifluoro-4-(hexyloxy)-4"'(octyloxy)-1,1':4', 1' -terphenyl [26], was chosen because it has multiple dipoles, which are therefore less likely to be orientated along the eigenvectors of its polarizability tensor. Optically measured phase transition temperatures for both materials are presented in Fig. 3.

In order to measure optical and dielectric properties of $\mathrm{X}$ and $\mathrm{Y}$, the two achiral materials exhibiting first-order $\mathrm{N}$ to Sm-C phase transitions were doped with $0.5 \%$ (w.r.t. mass) of chiral dopant BE8OF2N. This low concentration introduces spontaneous polarization $\left(\lesssim 5 \times 10^{-6} \mathrm{Cm}^{-2}\right.$ for the studied temperature ranges), which allows ferroelectric switching in the Sm-C* phase [13] while keeping the other physical properties the same as the undoped $\mathrm{Sm}-\mathrm{C}$ phase to within the experimental error range. Measurements were made by studying doped $\mathrm{X}$ and $\mathrm{Y}$ in (nominally) $2 \mu \mathrm{m}$-spaced planar $\left(2^{\circ}\right.$ surface pretilt) and homeotropic devices.

Such low concentrations of the chiral dopant also make the pitch much longer than the selected cell gaps, which allows the helix to be unwound by the planar-aligned surfaces, forming the surface stabilized state [15]. The samples were cooled from the isotropic phase at a rate of $1 \mathrm{~K} / \mathrm{min}$ using a Linkam T95-PE temperature controller with a resolution of $0.01 \mathrm{~K}$ and 
stabilized for $60 \mathrm{sec}$ before each set of measurements to achieve uniform alignment.

Polarized optical microscopy was used to find the director orientations in planar devices by observing optical extinction angles as a function of temperature. At each temperature increasingly stronger electric fields were applied as square waveforms with corresponding voltages from 0 to $10 \mathrm{~V}$ using the Aim-TTi TGA1241 arbitrary waveform generator. Following this, $\theta_{o}$ and $\delta$ were calculated from the infinite voltage asymptotes of the extinction angles [27,28].

After every $1 \mathrm{~K}$ interval, measurements of capacitance and dielectric loss taken with an Agilent E4980A precision LCR meter were performed on planar and homeotropic devices to find $\varepsilon_{p}$ and $\varepsilon_{h}$, respectively. Each set of measurements was taken by applying an ac voltage of $0.05 \mathrm{~V}$ at frequencies ranging from $20 \mathrm{~Hz}$ to $2 \mathrm{MHz}$. Subsequently, an additional dc voltage bias was applied at ascending magnitudes to the planar devices to find the infinite voltage asymptotes of capacitance to find $\varepsilon_{\mathrm{f}}$, given by Eq. (6).

Capacitance scans on Sm-C* liquid crystals can show two relaxation modes: due to field-induced fluctuations of the azimuthal director (Goldstone mode) at low frequencies and stretching with respect to the cones reference frame (soft mode) at higher frequencies. The latter occurred particularly close to the phase transition. Due to the first-order nature of $\mathrm{N}$ to Sm-C phase transitions, no soft mode was observed in $\mathrm{X}$ and $\mathrm{Y}$. On the other hand, a small degree of the Goldstone mode was expected at low frequencies, but ionic screening was found in practice. For this reason, a frequency of $10 \mathrm{kHz}$ was selected for calculating dielectric cone angles and principal permittivities of $\mathrm{X}$ and $\mathrm{Y}$, where no azimuthal fluctuations around the cone were observed.

\section{RESULTS AND DISCUSSION}

A comparison of optical and dielectric measurements for each compound confirms that there is a difference $\partial \theta$ between optical and dielectric cone angles. From Fig. 4 it is clear that $\partial \theta$ increases as the samples are cooled through the $\mathrm{Sm}-\mathrm{C}$ phases, showing an increasing degree of disassociation between the principal optical and permittivity axes. These results show that $\theta_{\varepsilon}$ may differ from $\theta_{o}$ by a considerable amount. As $\partial \theta$ increases, orthorhombic approximations become less appropriate for calculating the dielectric properties.

The introduction of monoclinic symmetry does not have a significant effect on the magnitude of dielectric anisotropy $\Delta \varepsilon$, which already exists in the uniaxial phase. However, dielectric biaxiality $\partial \varepsilon$ appears only in biaxial phases, which makes it sensitive to the monoclinic nature of the molecular shape. Since $\theta_{\varepsilon}<\theta_{o}$, using an orthorhombic approximation for a monoclinic molecule will result in an overestimated value of $\partial \varepsilon$. Consequently the switching times of ferroelectric Sm-C materials will show a varying degree of disagreement between the theoretical estimates and experimental values, depending on how monoclinic the phase is at a given temperature.

As expected, $\partial \varepsilon$ shows a positive correlation with reduced temperature for both $\mathrm{X}$ and $\mathrm{Y}$, as shown in Fig. 5. There is a first-order jump of $\partial \varepsilon$ at the $\mathrm{N}$ to Sm-C phase transition, which is followed by a roughly linear trend below a temperature slightly below the transition corresponding to the point at

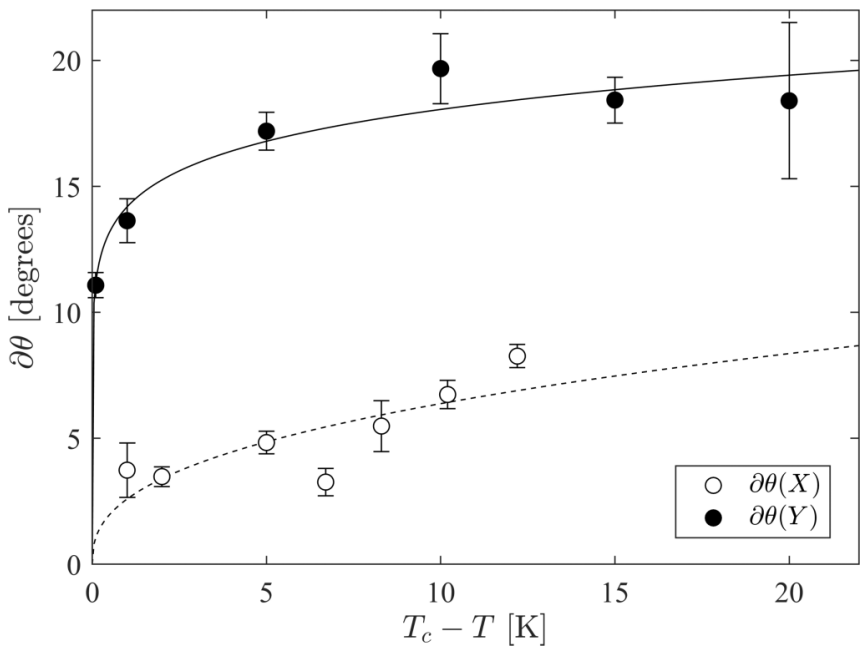

FIG. 4. Plot of the cone angle difference $\partial \theta$ against the reduced temperature below the $\mathrm{N}$ to $\mathrm{Sm}-\mathrm{C}$ phase transition $T-T_{c}$. Open symbols are used for $\mathrm{X}$, and closed symbols are used for Y. Lines are fits to $\partial \theta=a_{1}\left(T_{c}-T\right)^{a_{2}}$ and are used as guides for the eye only, where $a_{1}=2.6 \pm 0.9, a_{2}=0.39 \pm 0.16$ for $\mathrm{X}$ and $a_{1}=14.2 \pm 0.6$, $a_{2}=0.10 \pm 0.02$ for $\mathrm{Y}$.

which the layers become fixed at the device surfaces. Unlike $\mathrm{X}$, molecules of $\mathrm{Y}$ have several polar components, pointing in different directions, which contribute towards the overall transverse dipole moment. Thus, we observe higher $\partial \varepsilon$ in $\mathrm{Y}$ in comparison to those of $\mathrm{X}$. A comparison of $\partial \varepsilon$ to the results obtained from the orthorhombic approximation shows that such an approximation would result in an overestimation by more than a factor of two. However, it is also clear that this comparison is not appropriate, as the orthorhombic approximation does not allow the governing equations all to

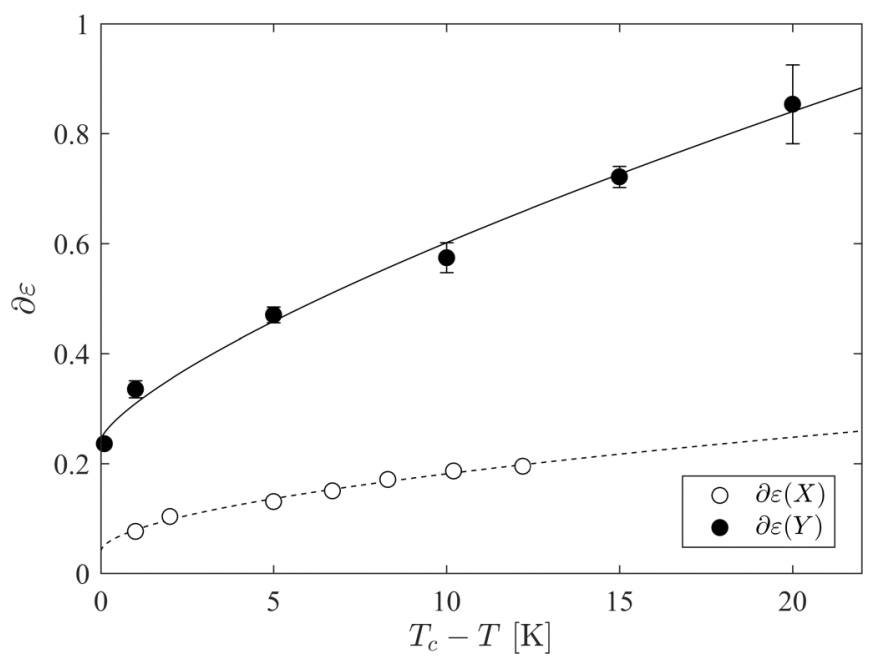

FIG. 5. Plot of dielectric biaxiality $\partial \varepsilon$ against the reduced temperature below the $\mathrm{N}$ to $\mathrm{Sm}-\mathrm{C}$ phase transition $T-T_{c}$. Open symbols are used for $\mathrm{X}$ and closed symbols are used for Y. Lines are fits to $\partial \varepsilon=$ $b_{1}\left(T_{c}-T\right)^{b_{2}}+b_{3}$ and are used as guides for the eye only, where $b_{1}=$ $(3.9 \pm 2.0) \times 10^{-2}, b_{2}=0.56 \pm 0.16, b_{3}=(3.9 \pm 2.4) \times 10^{-2}$ for $\mathrm{X}$ and $b_{1}=(6.6 \pm 2.3) \times 10^{-2}, b_{2}=0.74 \pm 0.11, b_{3}=0.24 \pm 0.03$ for $\mathrm{Y}$. 

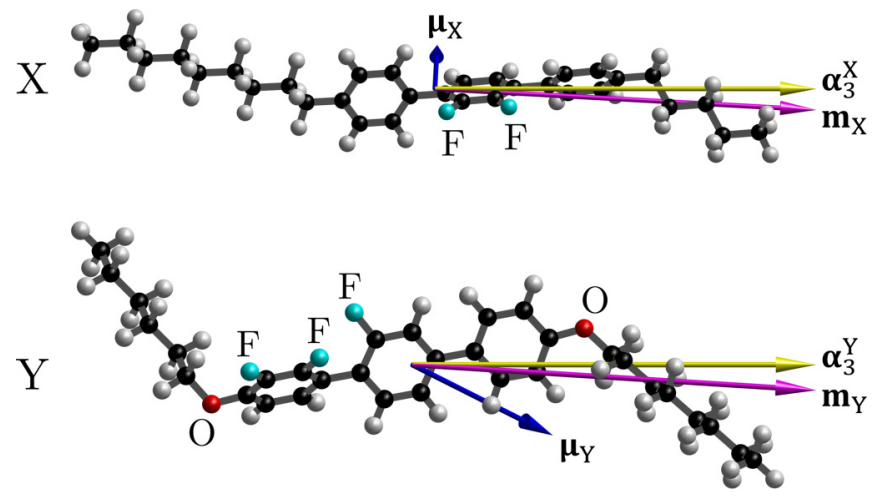

FIG. 6. Density functional theory B3LYP/6-31G(d) minimized energy configurations of $\mathrm{X}$ and $\mathrm{Y}$, where hydrogen, carbon, oxygen $(\mathrm{O})$, and fluorine $(\mathrm{F})$ atoms are represented by white, black, red, and cyan spheres, respectively (color online). Blue arrows show the direction of the net dipole moments given by $\mu_{X}=6.07 \times 10^{-30} \mathrm{Cm}$ for $\mathrm{X}$ and $\boldsymbol{\mu}_{Y}=9.84 \times 10^{-30} \mathrm{Cm}$ for $\mathrm{Y}$. Magenta arrows represent the average mass axis of each molecule $\mathbf{m}_{X}$ for $\mathrm{X}$ and $\mathbf{m}_{Y}$ for $\mathrm{Y}$. Yellow arrows represent the direction of the principal polarizability axis associated with the director $\boldsymbol{\alpha}_{3}^{X}$ for X and $\boldsymbol{\alpha}_{3}^{Y}$ for Y.

be satisfied within experimental error. Dielectric and optical measurements used for calculating $\partial \theta$ and $\partial \varepsilon$ can be found in the Appendix.

In this article, we have assumed that the optical director (indicated by $\mathbf{n}_{3}$ in Fig. 2) lies in the plane of the device. However, this is not necessarily the case if the mass axis does not coincide with the optical director. By simple consideration of the zigzag shape of the average molecule, we can deduce that the mass cone angle can be lower than $\theta_{o}$. By performing density functional theory simulations in GAUSSIAN 09 using B3LYP method and a 6-31G(d) basis set, while maintaining the general zigzag shape with a maximized transverse dipole moment to account for the Sm-C phase, we can see that the mass axis differs from the polarizability axes by $4.1^{\circ}$ for $\mathrm{X}$ (net dipole moment of $\mu=6.07 \times 10^{-30} \mathrm{Cm}$ ) and $4.6^{\circ}$ for $\mathrm{Y}\left(\mu=9.84 \times 10^{-30} \mathrm{Cm}\right)$ (see Fig. 6). The result is for individual molecules, which will be affected by the ensemble within the Sm-C phase and is valid only when there are no intermolecular interactions. Since the optic eigenvalues depend on polarizability, while the dielectric eignevalues also depend on dipole moment contributions, we expect that the mass axis will deviate from the optics as the cone angle increases. The analysis can also be performed with the assumption that the dielectric director (indicated by $\varepsilon_{3}$ in Fig. 2) lies in the plane of the device in the planar geometry. While the dielectric director is much farther away from the mass axis, it provides the other extremum to the solution set. The calculation requires an adjustment to the optical results and the principal permittivity values. From this, we see that $\theta_{o}$ and $\theta_{\varepsilon}$ remain within experimental error of each other, while $\delta$ has a significant decrease. Consequently, the angle measurements associated with monoclinic symmetry can be assumed to have negligible error generated from our assumption of the mass axis matching that of the optics. However, the effect on $\delta$ is more significant, which means that $\partial \varepsilon$ in Fig. 5 show the upper bound for the actual values. For this reason, it would be instructive to measure the layer tilt angle directly using $\mathrm{x}$ rays at a synchrotron source.

\section{CONCLUSIONS}

In conclusion, we have investigated monoclinic symmetry of Sm-C liquid crystals by showing that a disassociation can exist between the principal optical and dielectric axes with respect to one another. By applying invariance principles to the Sm-C phase, we can see that the eigenvector sets for optics and dielectrics must share at least one common axis, which must be parallel to the $C_{2}$ symmetry axis, due to monoclinic symmetry. The remaining axes need not coincide between optics and dielectrics, resulting in a disassociation of the two through a unitary rotation around the $C_{2}$ axis. This was interpreted as the existence of a dielectric cone angle $\theta_{\varepsilon}$ alongside the optic cone angle $\theta_{o}$. This resulted in a new variable, given by the difference between the two cone angles $\partial \theta$, which provides a geometrical
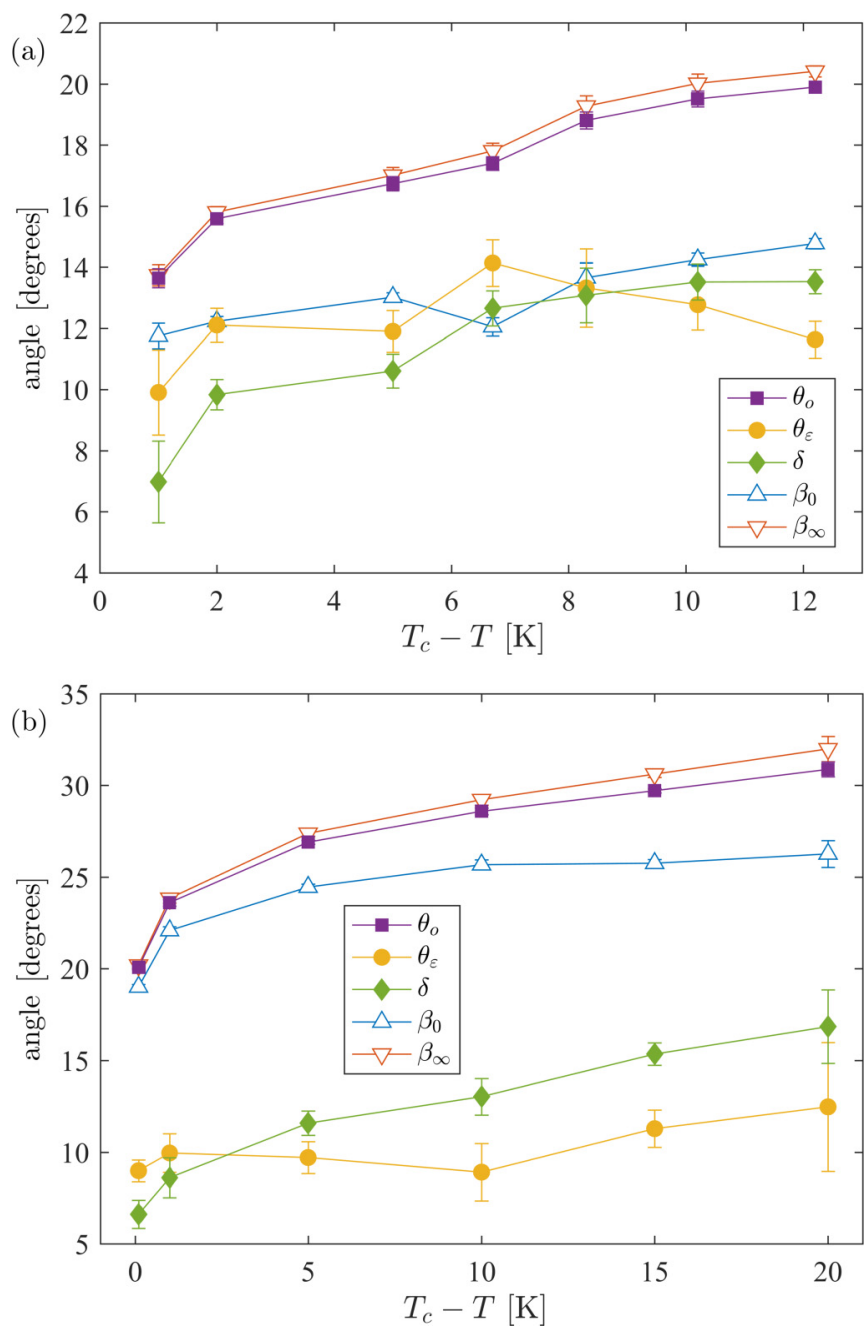

FIG. 7. Plot of optical cone angle $\theta_{o}$ (filled square), dielectric cone angle $\theta_{\varepsilon}$ (filled circle), layer tilt $\delta$ (filled diamond), zero voltage optical extinction angle $\beta_{0}$ (open upward pointing triangle), and infinite voltage optical extinction angle $\beta_{\infty}$ (open downward pointing triangle) against the reduced temperature $T_{c}-T$ for $\mathrm{X}$ (a) and $\mathrm{Y}(\mathrm{b})$. Lines serve as guides for the eye only. 

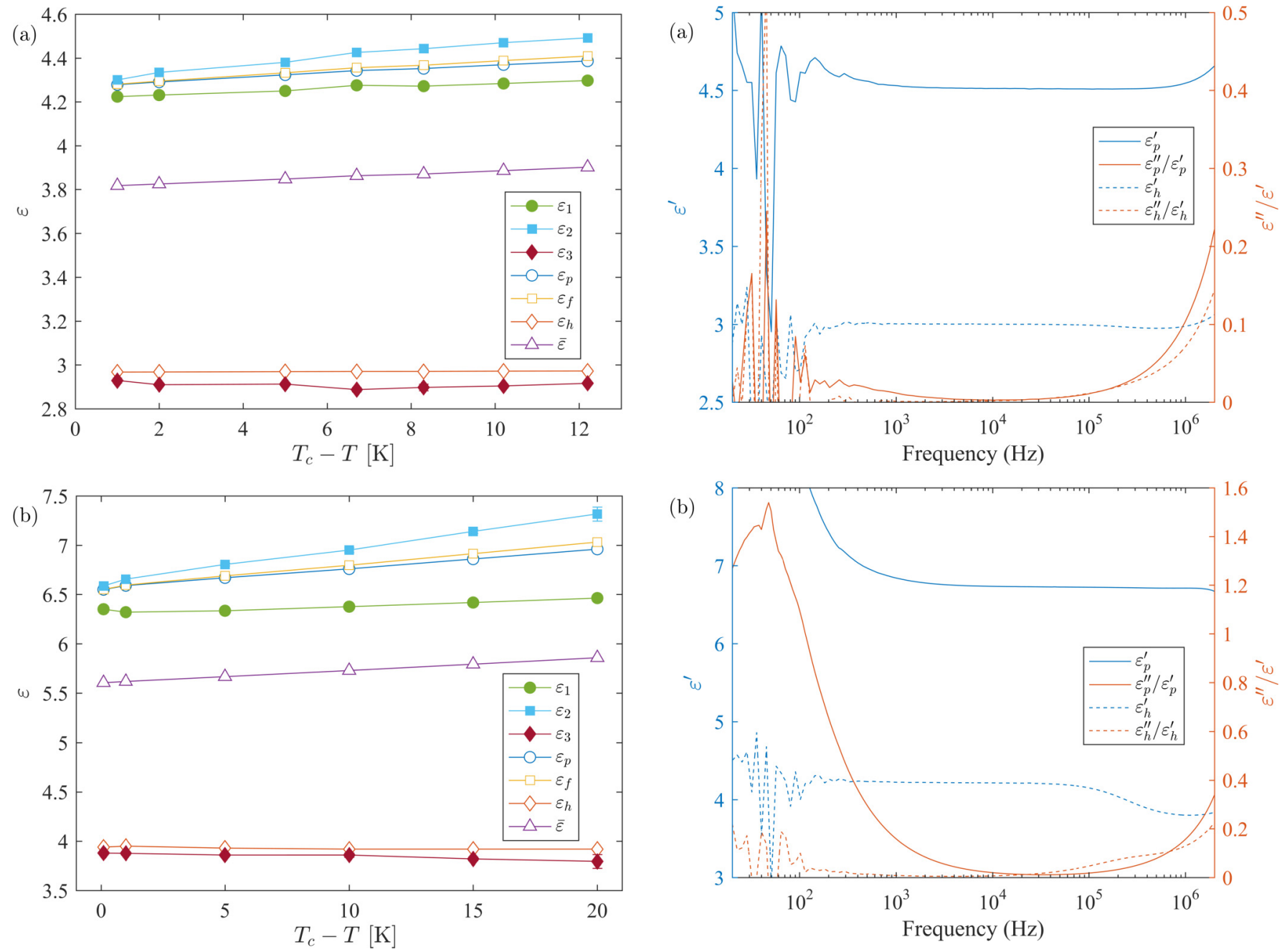

FIG. 8. Plot of principal permittivities $\varepsilon_{1}$ (closed circle), $\varepsilon_{2}$ (closed square), $\varepsilon_{3}$ (closed diamond), planar permittivity $\varepsilon_{p}$ (open circle), $\varepsilon_{f}$ (open square), $\varepsilon_{h}$ (open diamond), and $\bar{\varepsilon}$ (open triangle) against the reduced temperature $T_{c}-T$ for X (a) and Y (b). Lines serve as guides for the eye only. Dielectric data are shown for the temperatures corresponding to the optical measurements.

scale for monoclinic behavior. Following this, we have used two liquid crystal compounds with direct $\mathrm{N}$ to $\mathrm{Sm}-\mathrm{C}$ phase transitions to verify this hypothesis. Since the nature of the $\mathrm{N}$ to $\mathrm{Sm}-\mathrm{C}$ phase transition is first order, this resulted in highly pronounced monoclinic properties. The results indicated that $\partial \theta$ increased as the samples were cooled to as much as 20 for compound $\mathrm{Y}$ within the studied range of $20 \mathrm{~K}$ below the $\mathrm{N}$ to $\mathrm{Sm}-\mathrm{C}$ phase transition. On the other hand, $\partial \theta$ increased to only roughly half of that for compound $\mathrm{X}$. This suggests that multiple sources of dipole moment contributions result in a higher degree of monoclinic behavior. So far, other studies of ferroelectric liquid crystals have assumed $D_{2 h}$ symmetry for calculations of $\partial \varepsilon$, which resulted in overestimated $\partial \varepsilon$ and switching speeds. However, we have shown that monoclinic behavior becomes very important with decreasing temperatures and even immediately below first-order phase transitions. Therefore, this will be particularly important for future novel

FIG. 9. Plot of measured permittivities $\varepsilon^{\prime}$ (blue lines) and the corresponding dielectric losses $\varepsilon^{\prime \prime}$ (orange lines) for $\mathrm{X}$ (a) and $\mathrm{Y}$ (b) at $T_{c}-T=10 \mathrm{~K}$, where the solid lines represent the planar geometry, and the dashed lines represent the homeotropic geometry (color online).

devices, such as those using bent core liquid crystals, which are known to exhibit highly first-order behavior.

\section{ACKNOWLEDGMENTS}

The authors wish to thank Dr. M. Hird for synthesizing the materials. The authors also wish to thank the UK Engineering and Physical Sciences Research Council (EPSRC) and Merck for support through an iCASE studentship for N.V.S. J.C.J. also wishes to thank the EPSRC for support through an Advanced Fellowship (EP/L0154188/2). [29].

The data associated with this work can be found in reference

\section{APPENDIX: DIELECTRIC AND OPTICAL MEASUREMENTS}

To calculate the difference between the optical and dielectric cone angles $\partial \theta$ and subsequently the dielectric biaxiality $\partial \varepsilon$, 
a combination of optical and dielectric measurements were made.

Optical extinction angles of $\mathrm{X}$ and $\mathrm{Y}$ were measured as a function of applied dc voltage in $2 \mu \mathrm{m}$-spaced planar devices. The director was switched to both sides of the layer normal interchangeably to determine the orientation of the in-plane component of the layer normal. From this, zero voltage $\beta_{0}$ and infinite voltage asymptote $\beta_{\infty}$ extinction angles were calculated as a function of reduced temperature (both are measured in relation to the in-plane component of the layer normal). This allowed optical cone angles $\theta_{o}$ and layer tilts $\delta$ to be calculated numerically from

$$
\cos \delta=\frac{\cos \theta_{o}}{\cos \beta_{0}}=\frac{\tan \theta_{o}}{\tan \beta_{\infty}},
$$

which can be derived by considering the limiting cases for the relaxed and the fully switched orientations of the director $[27,28]$. Calculated values of $\theta_{o}, \theta_{\varepsilon}, \delta, \beta_{0}$, and $\beta_{\infty}$ from optical extinction angle measurements are shown as a function of reduced temperature in Figs. 7(a) and 7(b) for X and Y, respectively.

Optical measurements were combined with permittivity measurements in planar $\varepsilon_{p}$, fully switched planar $\varepsilon_{f}$, and homeotropic $\varepsilon_{h}$ geometries to find the dielectric cone angles $\theta_{\varepsilon}$ and the principal permittivities $\varepsilon_{1}, \varepsilon_{2}$, and $\varepsilon_{3}$ of $\mathrm{X}$ and $\mathrm{Y}$ by solving in Eqs. (6)-(9). Figures 8(a) and 8(b) show $\varepsilon_{1}, \varepsilon_{2}, \varepsilon_{3}$, $\varepsilon_{p}, \varepsilon_{f}, \varepsilon_{h}$, and $\bar{\varepsilon}$ as a function of reduced temperature for $\mathrm{X}$ and $\mathrm{Y}$, respectively.

Examples of broadband dielectric spectra at $10 \mathrm{~K}$ below the $\mathrm{N}$ to Sm-C phase transitions are shown in Figs. 9(a) and 9 (b) for $\mathrm{X}$ and $\mathrm{Y}$, respectively, where $\varepsilon^{\prime}$ represents the real component of dielectric permittivity and $\varepsilon^{\prime \prime}$ represents its imaginary component. By evaluating the dielectric loss factor $\varepsilon^{\prime \prime} / \varepsilon^{\prime}$ and $\varepsilon^{\prime}$, we can see that the static regime occurs around $10 \mathrm{kHz}$, which was selected for calculations of the main results.
[1] D. J. Gross, Proc. Natl. Acad. Sci. USA 93, 14256 (1996).

[2] L. M. Blinov, Structure and Properties of Liquid Crystals, Vol. 123 (Springer Science \& Business Media, New York, 2011).

[3] Z. Zhang, S. Kaur, B. Kundu, B. K. Sadashiva, and H. F. Gleeson, J. Mater. Chem. C 5, 1195 (2017).

[4] R. A. Reddy, C. Zhu, R. Shao, E. Korblova, T. Gong, Y. Shen, E. Garcia, M. A. Glaser, J. E. Maclennan, D. M. Walba et al., Science 332, 72 (2011).

[5] A. G. Vanakaras and D. J. Photinos, J. Chem. Phys. 128, 154512 (2008).

[6] C. Tschierske and D. J. Photinos, J. Mater. Chem. 20, 4263 (2010).

[7] T. Niori, T. Sekine, J. Watanabe, T. Furukawa, and H. Takezoe, J. Mater. Chem. 6, 1231 (1996).

[8] Y. Shimbo, E. Gorecka, D. Pociecha, F. Araoka, M. Goto, Y. Takanishi, K. Ishikawa, J. Mieczkowski, K. Gomola, and H. Takezoe, Phys. Rev. Lett. 97, 113901 (2006).

[9] G. R. Luckhurst, S. Naemura, T. J. Sluckin, T. B. T. To, and S. Turzi, Phys. Rev. E 84, 011704 (2011).

[10] P. K. Karahaliou, A. G. Vanakaras, and D. J. Photinos, J. Chem. Phys. 131, 124516 (2009).

[11] W. L. McMillan, Phys. Rev. A 8, 1921 (1973).

[12] A. Wulf, Phys. Rev. A 11, 365 (1975).

[13] P. G. de Gennes and J. Prost, The Physics of Liquid Crystals, International Series of Monographs on Physics (Clarendon Press, Oxford, 1995).

[14] R. B. Meyer, Mol. Cryst. Liq. Cryst. 40, 33 (1977).

[15] N. A. Clark and S. T. Lagerwall, Appl. Phys. Lett. 36, 899 (1980).
[16] L. A. Beresnev, V. G. Chigrinov, D. I. Dergachev, E. P. Poshidaev, J. Fünfschilling, and M. Schadt, Liq. Cryst. 5, 1171 (1989).

[17] J. C. Jones, Liq. Cryst. 42, 732 (2015).

[18] J. C. Jones and E. P. Raynes, Liq. Cryst. 11, 199 (1992).

[19] J. Hoffmann, W. Kuczynski, J. Malecki, and J. Pavel, Ferroelectrics 76, 61 (1987).

[20] F. Gouda, W. Kuczynski, S. T. Lagerwall, M. Matuszczyk, T. Matuszczyk, and K. Skarp, Phys. Rev. A 46, 951 (1992).

[21] T. P. Rieker, N. A. Clark, G. S. Smith, D. S. Parmar, E. B. Sirota, and C. R. Safinya, Phys. Rev. Lett. 59, 2658 (1987).

[22] L. D. Landau, E. M. Lifshitz, J. B. Sykes, and J. S. Bell, Electrodynamics of Continuous Media (Pergamon Press, New York, 1961), pp. 313-314.

[23] L. D. Landau, E. M. Lifshitz, J. B. Sykes, and M. J. Kearsley, Statistical Physics, 2nd ed. (Pergamon Press, New York, 1969), pp. 374-400.

[24] D. C. Lay, Linear Algebra and Its Applications, 4th ed. (Addison Wesley, Boston, 2012), pp. 395-397.

[25] M. Hird, Liq. Cryst. 38, 1467 (2011).

[26] M. E. Glendenning, J. W. Goodby, M. Hird, and K. J. Toyne, J. Chem. Soc., Perkin Trans. 2 0, 481 (1999).

[27] K. H. Yang, A. Lien, and T. C. Chieu, Jpn. J. Appl. Phys. 27, 2022 (1988).

[28] J. C. Jones, E. P. Raynes, M. J. Towler, and J. R. Sambles, Mol. Cryst. Liq. Cryst. 199, 277 (1991).

[29] N. V. Solodkov, M. Nagaraj, and J. C. Jones, Data associated with "Effects of monoclinic symmetry on the properties of biaxial liquid crystals" University of Leeds (2018), https://doi.org/10.5518/351. 